\title{
LA FENOMENOLOGÍA DE LA RAZÓN Y LA EXPERIENCIA ESTÉTICA. EdMUNd HUSSERL Y VASILY SESEMANN*
}

\author{
Phenomenology of Reason and Aesthetic Experience. \\ Edmund Husserl and Vasily SesemanN
}

\begin{abstract}
Resumen: La estética fenomenológica debería ser capaz de revelar cómo la estructura de cualquier objeto estético dado está conectada con la experiencia de ese objeto, así como demostrar las condiciones necesarias para la propia experiencia estética. Para hacerlo, hay que argumentar en contra de los supuestos unilaterales, como por ejemplo la suposición del objetivismo estético que postula la belleza como rasgo exclusivo de la realidad independiente del sujeto; o la creencia opuesta, que la belleza es esencial y únicamente la proyección del gusto subjetivo sobre las cosas en el mundo. Sesemann analiza el objeto estético y el acto estético, enfatizando su conexión. Esta conexión se refiere a lo que se describe en la fenomenología de Husserl como la correlación entre el objeto intencional y el acto intencional. Esta conexión puede ser descubierta sólo mediante el método fenomenológico: realizando la reducción fenomenológica. En este documento se explicará en primer lugar la percepción estética en la estética de Sesemann. Más adelante, se examina la concepción de la estructura del objeto estético en el contexto de la estética de Sesemann: la composición de los elementos, las sensaciones en relación con el significado, etc. Por último, el artículo sugiere que la estética de Sesemann se basa fundamentalmente en el método de la reducción fenomenológica.
\end{abstract}

Palabras clave: Fenomenología | Reducción fenomenológica | Estética | Descripción | Conciencia

\author{
Dalius Jonkus \\ Vytautas Magnus University, Lituania \\ phenolt@yahoo.com
}

\begin{abstract}
Phenomenological aesthetics should also be able to show how the structure of any given aesthetic object is connected with the experience of that object, as well as to demonstrate the necessary conditions for the aesthetic experience itself. In order to do so, one must argue against one-sided assumptions, such as the aesthetic objectivism's supposition that beauty is exclusively the trait of reality not at all dependent on the subject's experience of it; or its opposite belief that beauty is essentially and solely the projection of the subjective taste onto the things in the world. Sesemann analizes the aesthetic object and aesthetic act by emphasizing their connection. This connection relates to what is described in Husserls phenomenology as the correlation between the intentional object and the intentional act. This connection can be discovered only by using the phenomenological method: by doing phenomenological reduction. This paper will first explain the aesthetic perception in Sesemann's aesthetics. Later, it examine the conception of the aesthetic object's structure in Sesemann's aesthetic: composition of elements, sensations in connection with meaning; etc. Finally, the paper will argue that Sesemann's aesthetics is essentially based on the method of phenomenological reduction.
\end{abstract}

Key Words: Phenomenology | Phenomenological reduction | Aesthetics | Description | Consciousness

\footnotetext{
* Texto presentado en el X Congreso de la SEFE celebrado en Barcelona Fenomenología, experiencia y razón. En el Centenario de "Ideas I".
} 


\section{INTRODUCCIÓN}

La razón en la fenomenología de Husserl no es una cualidad del sujeto, sino una correlación entre el sentido de las cosas y su manera de aparecer en la conciencia. La racionalidad de la realidad exige una expresión adecuada. Esta correlación significa que la manifestación de las cosas no se da al azar, sino que es objetiva. La filosofía de la cultura y de la estética revela los significados culturales y estéticos intersubjetivos. El objetivo de esta ponencia es revelar la correlación entre la fenomenología de la razón y la experiencia estética, mediante la comparación de la fenomenología de Husserl y la estética de Vasily Sesemann. Vasily Sesemann nació en el año 1884 en la ciudad de Viborg, Finlandia. Su padre era sueco y su madre alemana. Estudió filosofía en la Universidad de San Petersburgo. De 1909 a 1911 continuó estudiando filosofía en la Universidad de Marburgo; en esa ciudad, junto con Ortega y Gasset, fue miembros del grupo de Nicolai Hartmann. En 1923 fue invitado a enseñar filosofía en la Universidad de Kaunas, Lituania. Durante la época del dominio de la Unión Soviética, fue arrestado y pasó seis años en el Gulag. Después de ser liberado se le permitió trabajar como profesor de lógica en Vilnius hasta el día de su muerte, el 23 de marzo de 1963. La filosofía de Sesemann fue influenciada por las ideas neokantianas y la fenomenología. Su teoría de la estética utiliza el método fenomenológico.

El interés de Sesemann en la estética se hace obvio no solamente por los temas tratados en su obra principal La Estética, publicada por primera vez en lituano en 1970, después de su muerte, sino también por las investigaciones hechas y publicadas en varios artículos. En el año 1922, en un artículo escrito en ruso y publicado en la revista rusa Pensamiento 1 durante la postrevolución en Petrogrado (actual San Petersburgo), Sesemann formula sus principios estéticos, que no cambiarán en sus publicaciones sucesivas ni en el manuscrito de su Estética. Es probable que su interés por la estética comenzara durante su época de estudiante en Marburgo, cuando, como hemos dicho, participa en el 
grupo de discusión de Nicolai Hartmann ${ }^{1}$. Para poder afirmar esta tesis es necesario analizar otras fuentes escritas como la correspondencia entre Sesemann y Nicolai Hartmann. Ahora podemos hacer conjeturas al respecto partiendo de las fuentes indirectas a disposición. José Ortega y Gasset, pensador que también pertenecía a este grupo de discusión, profundizó en varios aspectos de la estética y la filosofía de la cultura, reflexionando sobre las posibilidades de la aplicación de la fenomenología. Hartmann terminó de escribir su obra Estética, publicada en 1953, (que podemos definir como una estética fenomenológica) al final de la segunda guerra mundial 1944-1945. Todos estos pensadores mencionados mantienen una posición crítica con respecto a las teorías estéticas, tanto las psicológicas subjetivistas como las naturalistas objetivistas. Sesemann, como otros pensadores de su época, pone todo su interés en el libro Investigaciones lógicas de Husserl, en el que se explica el método fenomenológico y se anima a recurrir a la experiencia directa y a la descripción del objeto dado en ella. También representan un punto de atención para los intelectuales de este periodo las investigaciones de Moritz Geiger, que, partiendo desde un enfoque fenomenológico, intenta purificar el plano de la experiencia estética y revelar la singularidad de una vivencia estética. Sin embargo, los estudiosos de la estética de Sesemann no se han atrevido hasta ahora a realizar un análisis profundo de los vínculos de este autor con la tradición de la filosofía fenomenológica.

En este artículo se hace, en primer lugar, un análisis del objeto estético y su correlación con el acto estético; en segundo lugar, se muestra que las concepciones estéticas de Sesemann pertenecen a la tradición fenomenológica. En realidad la posición que el pensador sostiene con respecto a las ideas de Husserl sobre la fenomenología pura no está muy clara, debido a algunas circunstancias comprensibles. Aun así, yo considero que es obvia la conexión del desarrollo de su pensamiento con la fenomenología transcendental, si profundizamos en sus teorías sobre la estética, sobre todo al analizar el objeto estético o los valores en la correlación con el acto estético.

En un artículo de Javier San Martín publicado hace poco éste afirma que existen varias diferencias entre las Investigaciones lógicas y el primer tomo de

\footnotetext{
${ }^{1}$ Javier San Martín, en su libro dedicado a la fenomenología de Ortega y Gasset, menciona este grupo de Nicolai Hartmann. Cfr. San Martín, J., La fenomenología de Ortega y Gasset, Madrid, Biblioteca Nueva, 2012, pp. 52.
} 
las Ideas de Husserl. Entre estos dos libros se observa una cierta evolución relacionada con la nueva razón práctica y la comprensión del problema de los valores:

Si para considerar el tema del idealismo fenomenológico la distinción de estas dos etapas es fundamental, en la consideración de la razón práctica aún lo es más. De todas maneras, hay que ver una considerable evolución de Husserl ya en la etapa de la fenomenología estática, desde las Investigaciones lógicas a las Ideas. Pero me parece que, sobre todo, son más importantes los cambios posteriores. El cambio de las Investigaciones lógicas a las Ideas consiste en que en la primera obra los actos relacionados con la práctica, la valicepción y los sentimientos que motivan la voluntad, son meras cualidades intencionales a las que no se le podrían preguntar por su razón porque esta se vehicula solo con la materia intencional que es teórica y no tiene relación con la práctica. En las Ideas el análisis es mucho más matizado, porque los actos intencionales valiceptivos son actos noéticos que tienen sus correlatos en el noema, abriéndolos así a una fenomenología de la razón práctica. ${ }^{2}$

San Martín subraya que en el primer tomo de las Ideas, comparándolo con las Investigaciones lógicas, ocurre un cambio esencial al evaluar las posibilidades de la razón práctica; ésta se vincula, en primer lugar, al problema de los valores éticos. Sin embargo, yo creo que este giro hacia la práctica también nos revela el problema relacionado con los valores estéticos. La estética de Vasyli Sesemann es una teoria fenomenológica de los valores estéticos, mediante la cual se analiza y describe la objetividad de los valores estéticos. La fuente de esta objetividad es la correlación entre objeto estético y acto estético.

\section{DEFINICIÓN DEL OBJETO ESTÉTICO}

Según Sesemann, la estética es una disciplina que estudia el sentido del fenómeno estético. Los objetos creados por la naturaleza o por el ser humano que manifiestan su belleza pertenecen a la esfera de la estética. Sesemann ve en la estética una teoría de la belleza, esta, en su sentido más amplio, es iden-

\footnotetext{
2 San Martin Javier, "Valores y racionalidad en los diversos tipos de cultura", Escritos de Filosofia. Segunda Serie, Buenos Aires, No1, (2013), pp.117-118. 
tificada con el valor estético. Podemos decir que el objeto estético tiene su valor cuando es bello y esto supone que el objeto estético es dado al sujeto. La belleza como valor estético puede existir solamente como expresión sensorial para el sujeto que posee la cualidad de comprender y evaluar, es decir, que es capaz de reconocer el valor estético que contiene un objeto. En la misma definición del objeto estético reside la referencia a su asociación con una percepción particular y con el acto mismo de evaluación. Esto es un rasgo característico de la definición fenomenológica de los valores. Los valores normalmente sólo existen para aquellos que los aprecian y los reconocen. Sin embargo, lo importante no es sólo el acto de la intuición de los valores, sino también la estructura del objeto en sí mismo, que es un requisito previo para la realización del acto de evaluación. Sesemann hace hincapié en la relación existente entre los valores estéticos y el acto de evaluación: "Si la belleza es un valor determinado, que incluye obras de arte o de la naturaleza y los fenómenos de la vida humana, entonces no depende sólo de la naturaleza del objeto, sino también del sujeto, el cual al ser consciente del objeto, siente y reconoce su belleza" ${ }^{3}$.

Al considerar los criterios de la belleza estética, nos encontramos con la creencia común de que la belleza es una cuestión puramente subjetiva, o como dice un refrán popular "Sobre gustos no hay nada escrito". Esta actitud sugiere que la objetividad de la belleza se podrá descubrir sólo si es demostrada objetivamente mediante ciertos métodos experimentales, pertenecientes a las ciencias naturales y que además son independientes del sujeto. Sesemann condena tales puntos de vista e indica que la metodología de la investigación científica de los valores no puede coincidir con los métodos de las ciencias naturales, porque estaríamos negando los valores de los mismos fenómenos: "Exigir que la ética y la estética sigan los métodos de las ciencias naturales, significa hacer una investigación estética y ética de los fenómenos desde un enfoque que no permite el desarrollo y la captación de los rasgos característicos del fenómeno" ${ }^{4}$.

Otra crítica a la estética es que supuestamente es relativa. La belleza no es solamente algo subjetivo sino también relativo; cambia según las circunstancias culturales e históricas. Esta crítica como la primera enfatiza la belleza como un

\footnotetext{
${ }^{3}$ Sezemanas Vosylius, Estetika, Vilnius, Mintis, 1970, p. 12.

${ }^{4}$ Ob. cit., p. 14.
} 
valor dependiente del sujeto y de las circunstancias accidentales en la que se encuentra. Sin embargo, Sesemann rechaza este punto de vista porque, a su juicio, la objetividad aquí no significa necesariamente la negación del sujeto. La objetividad debe entenderse como la manifestación de la belleza ante el sujeto que percibe y valora, ya que dicha valoración debe reunir una serie de condiciones necesarias, como la estructura del objeto mismo y las correspondientes capacidades del sujeto. Por ejemplo, el estilo estético de una u otra época histórica no cambia por casualidad, sino de acuerdo a la relación cambiante del sujeto con el entorno también cambiante. Por lo tanto, podemos decir que esta relación o estilo es una unidad tan orgánica que incluso, aunque sufra cambios, puede definirse como constante. No es una coincidencia que Sesemann enlace la objetividad estética con la manera en que los fenómenos estéticos son dados al sujeto consciente de ellos y no con la objetividad misma y la realidad en sí del mundo existente.

El hecho de que la belleza se manifieste solo ante el sujeto que la percibe y aprecia, no implica inmediatamente que el acto de reconocimiento dependa por entero de las predilecciones individuales del sujeto, de su carácter y su parecer. Lo que depende de las condiciones subjetivas también puede ser objetivo, si esas condiciones se encuentran en la naturaleza general del sujeto o en sus relaciones esenciales con el objeto. En este sentido, en la cultura todos los valores son objetivos, a pesar de que no pueden existir sin el sujeto que los crea y percibe, mediante el cual pueden convertirse en objetos de conocimiento científico. ${ }^{5}$

La estética como ciencia no debería distanciarse del sujeto que percibe, del sujeto consciente, sino revelar las condiciones necesarias del fenómeno estético. Entonces la intención de la estética no es valorar ciertos fenómenos concretos, sino descubrir las condiciones transcendentales de la expresión estética. Esta consideración de las condiciones de expresión estética nos habla no solo de la naturaleza transcendental de la filosofía de este pensador, sino que también se refiere al vínculo que mantiene con la fenomenología de Husserl. El filósofo alemán en su libro Ideas relativas a una fenomenología pura (I tomo) hace de la reflexión transcendental el método más importante de la fenomenología y,

\footnotetext{
${ }^{5}$ Ibídem, p.13.
} 
de este modo, trata de evitar el objetivismo y el naturalismo inherente en las ciencias.

\section{OBJETIVIDAD DE LA PERCEPCIÓN ESTÉTICA}

Sesemann subraya que cualquier teoría estética se basa en las percepciones estéticas inmediatas. A medida que la percepción estética es valiceptiva, es decir, se centra en la belleza del objeto como un valor, está sujeta a las actitudes de la conciencia. Sin dichas actitudes específicas, sentir y constatar la belleza es imposible. No es suficiente observar el objeto que supone belleza. La apreciación de la belleza acontece en un acto de percepción especial, diferente a la percepción común. Sesemann indica que la experiencia estética es directamente accesible sólo al sujeto mismo que la vive, o sea que es alcanzable solo desde la perspectiva de la persona que la experimenta. Para los otros es factible manera indirecta a través de la expresión lingüística. ¿Significa esto que la experiencia estética está encerrada en la conciencia subjetiva? No. Según Sesemann la experiencia estética del otro es perceptible pero solamente cuando nosotros mismos la comprobamos partiendo de nuestra propia experiencia, o sea, al reproducir la misma percepción estética del otro. La intersubjetividad de la experiencia estética no sólo confirma la posibilidad de repetirla, sino también el hecho de que estas experiencias se basan en un fundamento objetivo: el mismo objeto estético. "Cuanto más se concentra la percepción en el mismo objeto, cuanto más siga las instrucciones inherentes a la estructura del objeto sin ceder a la arbitraria ficción de la fantasía subjetiva, mayor y más firme será la objetividad del contenido perceptual" ${ }^{\prime 6}$.

Sesemann evita hacer subjetiva o relativa una experiencia estética, porque explica que el fundamento de dicha experiencia es el mismo objeto estético con su estructura, o sea el objeto intencional. Para que el sujeto pueda percibir un objeto estético, es necesario cumplir con ciertos requisitos. El sujeto no puede percibir un objeto de cualquier forma, esta percepción depende de la estructura del objeto dado en la experiencia. En otras palabras, la disponibilidad de la intersubjetividad de la vivencia estética está garantizada por la posibilidad de

\footnotetext{
${ }^{6}$ Ob. cit., p. 19.
} 
repetir ese acto y revivirlo como propio; la repetición sólo es posible debido a que el acto mismo va dirigido al mismo objeto, que por la expresión de su estructura proporciona instrucciones al sujeto que vive la experiencia y no permite la comprensión arbitraria.

En su artículo "La naturaleza poética de la imagen" (1925), Sesemann hace una análisis fenomenológico de las singularidades de la estructura de la imagen poética, como se manifiestan directamente en el lenguaje de la expresión poética. Él se basa en la suposición de que la estética en primer lugar se fundamenta en la experiencia directa, la cual es imposible de reducir al sujeto o al objeto, sino que se compone de los dos, formando un todo. La estética no solo trata de describir psicológicamente las vivencias internas, sino que concentra su atención en el fundamento objetivo de esas vivencias. Sesemann describe la correlación entre la experiencia intencional y el objeto intencional. Esta correlación es paradójica porque incluye sujeto y objeto al mismo tiempo. Además esta correlación abarca lo dado físico sensorialmente y la experiencia del sentido. Así que la realidad estética sólo se puede revelar a través de la experiencia estética enfocada hacia el objeto estético trascendental.

Sin embargo, Sesemann revela que tal percepción estética, en la que se garantiza la objetividad a través de la correlación entre el objeto y la experiencia, conlleva ciertos riesgos. En primer lugar, la objetividad estética o sea, la evaluación de la expresión estética sólo puede lograrse mediante la formación y la disciplina. Además, la percepción estética puede ser falsificada:

La falsificación proviene de dos fuentes. Primera [...] las limitaciones del mismo sujeto, la estrechez de su visión espiritual, su tendencia a interpretar todo de acuerdo a esquemas habituales y a su peculiaridad e individualidad. $Y$ la segunda es una imitación o mimetismo, que ha desempeñado un papel importante tanto positivo como negativo en la vida social. Cuando evaluamos algo, a menudo no lo hacemos de forma independiente, no nos basamos en nuestras propias experiencias, sino que seguimos los ejemplos de otros, estamos orientados hacia nuestro entorno social, la opinión pública o ciertas autoridades. ${ }^{7}$

Así como cualquier otra experiencia cultural, también la experiencia estética puede ser falsificada, ya que en lugar de la experiencia directa de valores, pue-

\footnotetext{
${ }^{7}$ Ob. Cit., p. 19.
} 
den aparecer exigencias o necesidades para cumplir con el prestigio social o la moda. En este caso, la persona no solamente engaña a los demás, sino también a sí mismo. La única solución para evitar la falsificación de la experiencia estética es perseguir la verdad de la realidad estética. Y la única persona que puede lograrlo, según Sesemann, es "sólo aquella que constantemente comprueba y profundiza su propia experiencia" ${ }^{\prime 8}$.

\section{LAS SENSACIONES Y EL SENTIDO EN LA PERCEPCIÓN ESTÉTICA}

Cuando hablamos sobre la expresión de los objetos estéticos se suele destacar el papel de la expresión sensorial. Los objetos estéticos no podrían existir sin la expresión sensorial. Por lo tanto la dimensión estética se asocia a la participación directa en la expresión sensorial. Sesemann muestra dos cosas: primero, que la expresión sensorial es inseparable del sentido y en segundo lugar, que la conexión entre la expresión sensorial y el sentido es ambigua; porque no es el sentido el que forma la materia sensorial, sino al contrario, la misma materia sensorial da forma al sentido. Primero, Sesemann hace una crítica a la noción dualista de la expresión sensorial y del sentido, a la expresión externa que se contrapone al sentido interno. Un ejemplo obvio es cualquier expresión humana. Si queremos entender la vida espiritual de una persona no es necesario recurrir a un razonamiento por analogía, como sostienen las teorías tradicionales. Sesemann, parte de las descripciones fenomenológicas de Scheler y dice:

\footnotetext{
A partir de las palabras, gestos, y mímica, yo percibo inmediatamente los pensamientos, sentimientos, deseos, estados de ánimo, etc., del otro; y esta percepción (y entendimiento) para mí como sujeto que percibe, son tan reales y objetivos como cualquier otra percepción. Los errores y equivocaciones que se producen aquí son los mismos que en la percepción del mundo material. ${ }^{9}$
}

Sesemann establece un paralelo entre la percepción de las expresiones estéticas y la percepción de las expresiones humanas. La expresión se caracte-

\footnotetext{
${ }^{8}$ Ibidem.

${ }^{9}$ Ibidem, p.331.
} 
riza por el hecho de que el sentido vive en ella y no más allá de ella. Por lo tanto, la expresión no tiene que ser conocida a tal punto como si quisiéramos reconstruir el otro lado de los significados arraigados. La representación estética destruye la oposición supuesta entre lo corporal y lo espiritual, entre el material sensorial y el sentido. Sesemann señala que, en la estética, la empatía juega un papel muy importante. Según este filósofo, la empatía no puede ser explicada desde la analogía o desde el concepto de la proyección psicológica; porque estos dos métodos suponen una distinción radical entre el sujeto y el objeto, además de una oposición entre ellos. Sesemann argumenta que la empatía es fundamental para la percepción estética porque se basa en la correlación entre las formas expresivas del mundo y la percepción incorporada.

No menos importante es que el material sensorial en el arte compone el medio de expresión. El análisis del lenguaje poético hecho por Sesemann demuestra que en la poesía la palabra se eleva a un primer plano como elemento sensorial. La palabra misma como expresión sensorial adquiere valor estético, ya que no es solo herramienta de representación, sino también un objetivo. Este razonamiento sobre la palabra poética y la expresión ritmico-sensorial del sentido recuerda el análisis posterior hecho por Heidegger en el que sostiene que la palabra poética revela la esencia del lenguaje. El punto es que la palabra poética se convierte en el objetivo de la expresión del sentido lingüístico. El significado de la palabra coincide con el ser sensorial de la palabra, porque en el lenguaje poético "la composición rítmico sonora de la palabra se transforma en una realidad tangible y obtiene un valor por sí misma" (Sesemann, 1970, 335). Esta coincidencia entre el sentido y la expresión sensorial puede ser diferente en las distintas formas del lenguaje. Por ejemplo, en el lenguaje artificial y en el lenguaje cotidiano no nos damos cuenta de esta coincidencia, pero en el lenguaje retórico la expresión sensorial adquiere una gran importancia. Solo en el lenguaje poético la palabra es mágica porque la expresión sensorial coincide con el sentido. (Sesemann, 1970, 34).

En su Estética, Sesemann no solo analiza el lenguaje poético para describir la relación entre la expresión sensorial y el sentido, sino también la pintura y la música. En la pintura es más importante la organización sensorial de los colores y las líneas que el tema representado. En general, el tema puede faltar pero sin colores y líneas, de acuerdo a Sesemann, la pintura no puede ser: 
Por lo tanto, es importante no sólo que de la misma imagen, o sea que del conjunto de colores y líneas que cubren una superficie, quede claro lo que significa y representa una pintura ( $y$ no de las inscripciones del cuadro), sino ante todo que el tema (del objeto representado) surja de la propia belleza representada (de la percepción sensorial) y sea justificado por esta. La percepción estética en comparación con una normal (no estética) es de dirección inversa: un pequeño instante del tema está determinado por los elementos sensoriales. ${ }^{10}$

Para Sesemann la percepción estética es lo contrario a la percepción normal (no estética), porque en la percepción normal el tema está condicionando la aparición de los elementos sensoriales (colores, sonidos, formas etc.) y su estructura, pero en la percepción estética los elementos sensoriales son el fundamento del tema porque el significado del mismo se ajusta a ellos. En la música, así como en el lenguaje poético y la pintura, Sesemann descubre la misma coincidencia: la existente entre los elementos sensoriales y el sentido de expresión. En la música, el rol de la organización sensorial es más evidente. La poesía y la pintura están atadas a la representación temática de los objetos, la música en cambio no representa nada, sino que expresa directamente los sentimientos y la dinámica interna de la experiencia.

En sus dos libros, las Investigaciones lógicas y en las Ideas relativas a una fenomenología pura y una filosofía fenomenológica, Husserl hace la distinción entre la materia sensorial y la forma intencional. Él argumenta que la materia sensorial primaria adquiere forma solo a través de los actos intencionales; es decir, que el material sensorial sin el acto intencional no tiene sentido. Sin embargo, Wilhelm Schapp en su Fenomenología de la percepción así como otros fenomenólogos hacen énfasis en la existencia de una correlación entre la experiencia sensorial y el sentido intencional.

Al profundizar en lo específico de la percepción estética, Sesemann sostiene que esta percepción se caracteriza por la orientación al todo como unidad de las partes. En otras palabras, nuestra experiencia no se compone de fragmentos sensoriales a los cuales después se les adjudican un significado, sino al contrario, el ser humano percibe los objetos como un todo. La estructura de cada objeto no solo se compone de fragmentos sensoriales sino se expresa como totalidad en la que cada elemento es una parte esencial del conjunto.

\footnotetext{
${ }^{10}$ Ibidem, p.36.
} 
Muchos fenomenólogos comparten la idea de la comprensión de la percepción como el entendimiento de un todo. Así explican la percepción Hartmann y Merleau-Ponty. Sesemann va un paso más allá y desarrolla esta idea. Sostiene que el acto de percepción está compuesto no solo de las impresiones directas sino que está relacionada con la experiencia acumulada. En el momento en que tiene lugar la percepción, los esquemas de comprensión organizan los elementos sensoriales y su significado. Los esquemas de comprensión permiten complementar los datos y organizarlos en una dirección determinada. Sesemann afirma que "en cada percepción sensorial, aun si es verdadera o falsa, nosotros percibimos más de lo que sentimos" ${ }^{11}$. Los esquemas de la experiencia descritos por Sesemann no son otra cosa que la descripción que hace Husserl de los mecanismos de la constitución. El significado de un objeto como constitución del todo no exige repetir cada vez la secuencia de operaciones que permite esquemáticamente constituir uno u otro significado.

\section{LA ESTRUCTURA DEL OBJETO ESTÉTICO}

Sesemann en su Estética analiza tanto la percepción estética como el objeto estético. La estética es impensable sin el análisis del objeto, porque la objetividad de la percepción estética surge del mismo objeto. Nicolai Hartmann, en su Estética, también hace hincapié en el análisis de la estructura del objeto estético. Dice: "Los valores estéticos: la belleza y su diversidad, son inherentes a la estructura del objeto, es decir a su totalidad multidimensional. Empezando desde el análisis del acto vamos hacia al análisis de la estructura del objeto, pues solo analizando la estructura del objeto podemos realizar el análisis del valor"12.

Este filósofo centra el análisis de la estructura del objeto estético en la relación entre el primer plano y el fondo, después pasa a la descripción multidimensional del objeto estético.

Si hablamos del problema de la estructura del objeto estético, observamos que en el análisis de Sesemann es más articulado y se desarrolla un tanto dife-

\footnotetext{
${ }^{11}$ Ibidem, p.26.

12 Hartmann Nicolai, Ästhetik, Berlin, Walter de Gruyter, 1966, pp.82.
} 
rente que en la filosofía de Hartmann. En primer lugar, cabe señalar que Sesemann enfatiza no solo la naturaleza intencional del objeto estético, es decir, su dependencia de la percepción estética, sino también la dependencia inversa; argumenta que la unidad sensorial del objeto estético es necesaria, porque si se desvía la intención de comprensión hacia las partes, el acto de comprensión pierde su carácter estético. Tal correlación entre la estructura del objeto estético y la percepción estética es mucho más importante para Sesemann que para Hartmann.

Analizando la estructura del objeto estético Sesemann distingue la unidad del objeto estético, el significado de los componentes, la unidad estática y genética y la expresión espacial y temporal. La unidad del objeto estético es un todo con diferentes componentes. Estos componentes son elementos diferentes que solamente al formar una unidad, un todo, pueden llegar a expresar un vaIor estético. Cada expresión artística presenta sus propios componentes; es el componente el que varía dependiendo de la naturaleza del arte; en la música están los tonos e intervalos, en la poesía las palabras, en el dibujo los contornos $y$, en la pintura, los colores y la luz; y en todas las artes existe una dinámica rítmica como componente imprescindible para la expresión. Estos elementos forman un todo, son parte de una unidad manteniendo una relación opuesta entre ellos, una relación de equilibrio o una relación simétrica.

La singularidad del objeto estético depende de los elementos distintivos y de la relación entre los componentes que lo forman. Pero la totalidad del objeto estético se forma no sólo por la combinación de diferentes elementos, sino también haciendo una distinción de los elementos que deben dominar. El elemento principal en la unidad del objeto estético es llamado por Sesemann factor dominante. De este factor depende el primer y segundo plano del objeto estético $y$, por supuesto, su composición general. Es decir, la composición de los elementos del objeto está imponiendo la lógica de la aparición para sujeto.

\section{CONCLUSIONES}

Sesemann analiza el objeto y el acto estéticos dándole una importancia clave a la relación existente entre ellos. La relación entre el objeto estético y el acto estético corresponde a la descripción de la correlación entre objeto inten- 
cional y acto intencional en la fenomenología de Husserl. Esta relación puede ser descubierta sólo mediante el uso del método fenomenológico: la reducción fenomenológica. Para llevarla a cabo, hay que neutralizar la suposición objetivista que afirma que la belleza es un rasgo de la realidad independiente del sujeto y superar la creencia subjetivista de que la belleza es la proyección del gusto subjetivo en objetos reales. La descripción fenomenológica revela la belleza como la relación de los valores con la intuición del sujeto evaluador y la disposición especial. La reducción que realiza Sesemann le lleva a reflexionar sobre las condiciones primarias de la expresión del objeto estético, presentes tanto en la estructura del objeto estético como en la disposición del sujeto que obedece a las exigencias de belleza del objeto. Este filósofo subraya que la objetividad de los valores estéticos no niega la participación del sujeto. La objetividad es nada menos que la lógica de la expresión del objeto estético. Es por eso que cada dimensión de la expresión estética se caracteriza por una unidad única del material sensorial y el sentido. La fenomenología de la razón estética es posible porque los actos intencionales valiceptivos son actos estéticos que tienen sus correlatos en la estructura de los objetos estéticos. 